\title{
International guidelines for prevention and management of post-operative chronic pain following inguinal hernia surgery
}

\author{
S. Alfieri · P. K. Amid • G. Campanelli • \\ G. Izard · H. Kehlet · A. R. Wijsmuller • \\ D. Di Miceli · G. B. Doglietto
}

Received: 18 October 2010/ Accepted: 28 January 2011/Published online: 2 March 2011

(C) Springer-Verlag 2011

\begin{abstract}
Purpose To provide uniform terminology and definition of post-herniorrhaphy groin chronic pain. To give guidelines to the scientific community concerning the prevention and the treatment of chronic groin and testicular pain.

Methods A group of nine experts in hernia surgery was created in 2007. The group set up six clinical questions and continued to work on the answers, according to evidencebased literature. In 2008, an International Consensus Conference was held in Rome with the working group, with an audience of 200 participants, with a view to reaching a consensus for each question.
\end{abstract}

S. Alfieri $(\varangle) \cdot$ D. Di Miceli · G. B. Doglietto

Department of Digestive Surgery, Catholic University of the Sacred Heart, Largo A. Gemelli, 8, 00168 Rome, Italy

e-mail: s.alfieri@rm.unicatt.it

P. K. Amid

Department of Surgery, UCLA Medical Center, Lichtenstein Hernia Institute at University of California Los Angeles,

Los Angeles, CA, USA

G. Campanelli

Department of Surgery, University of Insubria, Varese, Italy

G. Izard

Service de Chirurgie Digestive, Centre Hospitalier de Bigorre, Boulevard de Lattre de Tassigny, Tarbes Cedex, France

H. Kehlet

Section for Surgical Pathophysiology, Rigshospitalet

Copenhagen University, Copenhagen, Denmark

A. R. Wijsmuller

Department of Surgery, Erasmus Medical Centre,

University Medical Centre, Rotterdam, The Netherlands
Results A consensus was reached regarding a definition of chronic groin pain. The recommendation was to identify and preserve all three inguinal nerves during open inguinal hernia repair to reduce the risk of chronic groin pain. Likewise, elective resection of a suspected injured nerve was recommended. There was no recommendation for a procedure on the resected nerve ending and no recommendation for using glue during hernia repair.

Surgical treatment (including all three nerves) should be suggested for patients who do not respond to no-surgery pain-management treatment; it is advisable to wait at least 1 year from the previous herniorraphy.

Conclusion The consensus reached on some open questions in the field of post-herniorrhaphy chronic pain may help to better analyze and compare studies, avoid sending erroneous messages to the scientific community, and provide some guidelines for the prevention and treatment of post-herniorraphy chronic pain.

Keywords Hernia - Chronic pain .

Consensus conference $\cdot$ Nerve handling

\section{Introduction}

Persistent post-herniorrhaphy pain is increasingly recognized, but much controversy still exists in the literature regarding its incidence, terminology, pathogenesis and treatment strategies.

With the aim of analyzing these problems and to try to provide guidelines to the scientific community, a working group of nine international experts was created in 2007, selected on the basis of experience and their track records of publications in this field. 
Table 1 List of clinical questions

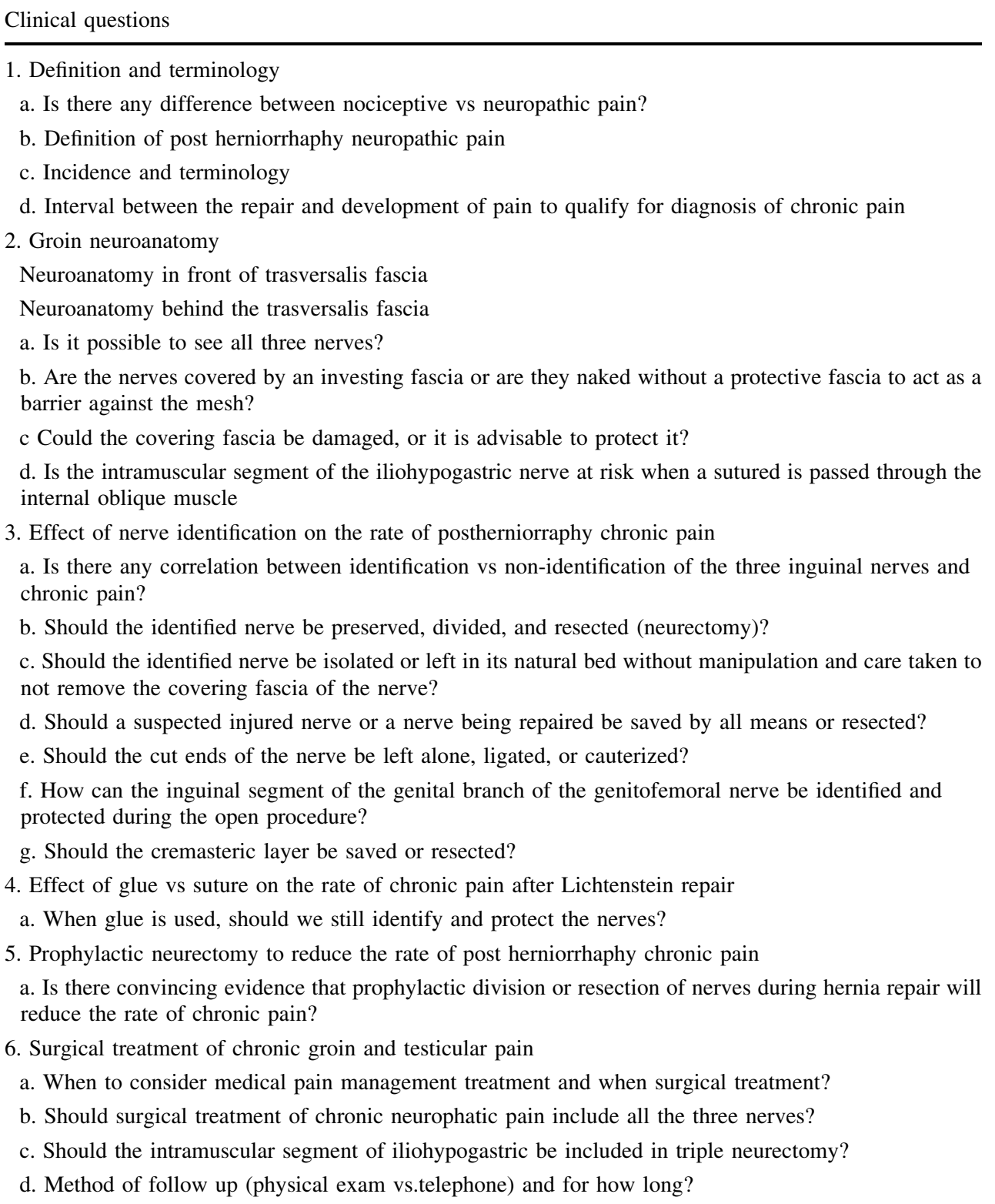

The working group set up six clinical questions with 23 subdivisions (Table 1) and continued to work for 1 year on the answers, according to the current evidence-based literature. Analyzing the literature, the panel assessed not only the level of evidence (LE) but also the grade of recommendations (GR) in each published study cited. They also examined each article, reading the entire paper, evaluating its statistical validity and appropriateness of the terminology used.

A meeting on these topics was held in Rome on the 21-22 April 2008 at the Catholic University of the Sacred Heart. The Consensus Conference was organized in two sections: the first was a closed session reserved for the working group, who had tried to reach a consensus for each of the six questions studied during the previous year. The second session was open to an international audience of about 200 participants. During this session, the working group repeated their corresponding presentations. Subsequently, the audience gave their answers (by electronic vote) to the statements prepared by the working group after each presentation, consisting of a review of the current literature for every single question.

The results that emerged from both the working group and the international consensus meeting with the audience represent the proposed guidelines at this moment in time.

The Consensus Conference was held under the aegis of the Italian Chapter of the European Hernia Society and the Italian Surgical Society. 


\section{Definition and terminology}

The overall literature is somewhat confusing regarding the definition of neuropathic pain, and hence the assessment of pain and its consequences in most trials have not been well defined [7, 20, 29, 32, 48, 49]. More recently, several questionnaires and validated score systems have been presented, concluding that pain should be assessed at rest as well as during well-defined functions corresponding to daily life tasks, including questions about pain influencing sexual function like dysejaculation [26, 31, 43, 58, 61].

Is there any difference between nociceptive vs neuropathic pain?

The current definition of neuropathic pain is a pain caused by direct nerve injury [60] characterized by various types of sensory dysfunction (hyperalgesia, hypoesthesia, allodynia, etc.) in the surgical area. This differs from nociceptic pain, which is due to tissue injury or an inflammatory reaction [2]. However, recent studies in postherniotomy patients without pain have also demonstrated the significant occurrence of sensory disturbances compared to the un-operated side [4], indicating that, during both open and laparoscopic groin hernia repair, most patients develop some type of nerve lesion with secondary sensory disturbances, but that only a proportion (probably around 10\%) will lead to a chronic pain [32]. Although preoperative pain may be related to the risk of developing chronic pain, there are no signs of preoperative sensory dysfunction in patients with or without pain [3]. Also, it should be emphasized that symptoms and signs in suspected neuropathic pain are very unspecific [56], and previous suggested classification of post-herniorrhaphy pain syndromes [40] requires further documentation of validity and further discussion of the consequences for the choice of treatment.

Obviously, a groin hernia repair with a mesh implant can lead to chronic nociceptive pain due to the continuous inflammation around the mesh. However, so far no study has clearly differentiated neuropathic pathogenesis from inflammatory pathogenesis, since the mesh inflammation by itself may lead to nerve damage. Therefore, studies are in progress based on well-defined neurophysiological assessments to identify sub-groups with different types of neuroplasticity (wind-up phenomena, allodynia, reduced pressure pain thresholds, hypoesthestia, etc.), which hopefully will be able to identify patients who can be reoperated $[5,6]$ versus those requiring pharmacological treatment [30].

In short, at present we are unable to clearly differentiate chronic neuropathic from nociceptive pain following groin hernia repair, although nerve damage seems to be a prerequisite for development of chronic pain [32, 37].

Definition of post herniorrhaphy neuropathic pain

The International Association for the Study of Pain (IASP [11]) defines chronic pain as a pain lasting for $>3$ months after the injury. Most papers use this definition for postoperative herniorrhaphy chronic pain; however, this definition has been based on non-surgical chronic pain.

In an attempt to unify the terminology, the working group, according also to the IASP definition, decided on the following definition for chronic post herniorrhaphy neuropathic pain: a pain arising as a direct consequence of a nerve lesion or a disease affecting the somatosensory system, in patients who did not have groin pain before their original hernia operation, or, if they did, the post-operative pain differs from the pre-operative pain.

A present, clinical diagnosis of neuropathic pain is not well defined.

Incidence and terminology

Regarding the incidence of chronic pain, the data present in literature are pretty heterogeneous: this variance $(0.7-43.3 \%)$ is due to differing definitions of chronic pain, different times of assessment and different methods of measurement. However, reviewing the literature [36], the working group agreed to a prevalence of $0.5-6 \%$ for the rate of debilitating pain affecting normal daily activities or work.

Another important issue was the need to provide a uniform terminology to be used in this field. In fact, the terms "division", "resection", "dissection", "transection", "section" and "neurectomy" are often used and reported wrongly, influencing and distorting the real results of studies, with important and practical implications regarding the treatment of chronic pain [12]. A clear example of this aspect is, for instance, the incorrect interpretation of Picchio' s paper [54], which is often cited as a study demonstrating that pain is not affected by elective division of the ilioinguinal nerve, whereas, reading the whole paper, it is possible to find out that the author actually performed a neurectomy (dividing the nerve lateral to the internal ring) instead of a simple division of the nerve in the operative field. Consequently, the results of this paper have often been distorted, which may also give the erroneous message that the nerves may be sectioned during operation at any level of the inguinal canal without any consequence [10].

With the aim of providing a uniform terminology, the working group decided to recommend the following nomenclature: 
- Cutting or dividing a nerve means interrupting the continuation of a nerve.

- Resection of a nerve or neurectomy means removing a segment of a nerve along the inguinal canal.

It is also appropriate to mention the handling of the cut ends (ligation, cauterization or nothing) of the proximal and distal residual nerve.

Interval between the repair and development of pain to qualify for a diagnosis of chronic pain

Prospective detailed studies in groin hernia repair have shown a predominantly continuous development of persistent pain, since patients who have significant pain 1 and 4 weeks postoperatively have a three- to ten-fold higher risk, respectively, of continuing into persistent pain [23]. There is less detailed information on the frequency and mechanisms of developing chronic post-herniotomy pain after a "pain-free" period. Finally, the question of "burnout" of a chronic pain syndrome has been assessed in a nationwide series $[1,20]$, where, at 6 years postoperatively, the incidence of pain influencing daily activities decreased by about $50 \%$ [1]. More studies are required in the same well-defined patient population to identify the incidence and mechanisms for developing a "new" chronic pain after an initial pain-free period.

To provide the scientific community with a provisory definition, the working group suggests that, for inguinal chronic pain only, the IASP [26] definition be modified from 3 months after surgery to more than 6 months after hernia surgery.

\section{Groin neuroanatomy}

Neuroanatomy in front of trasversalis fascia

The inguinal canal is crossed by three sensory inguinal nerves: the ilioinguinal, iliohypogastric and genital branch of genitofemoral nerve [38, 63].

Neuroanatomy behind the transversalis fascia

Behind the transversalis fascia the preperitoneal space is divided into two compartments by a septum. According to one school [16], this septum is the deep layer of the transversalis fascia, while another school [44], based on embryology, states that it is independent of the transversalis fascia. Regardless of the source, there are two compartments in the preperitoneal space: the parietal compartment between the transversalis fascia and the septum and visceral compartment between the septum and the peritoneum. The visceral compartment contains the bladder and prostate.

\section{Is it possible to see all three nerves?}

Some authors think that nerve identification during open hernia surgery could influence the incidence of postoperative chronic pain [13, 17, 35]. However, it is not clear if it is always possible to identify all three nerves, and this issue has not received due attention. According to the Netherlands Hernia Registry, only $32 \%$ of surgeons identify the iliohypogastric nerve during hernia repair, and only $36 \%$ identify the genital branch of the genitofemoral nerves [64].

Looking at the literature, only two anatomical studies have investigated the presence and course of the three inguinal nerves together [55, 64], while others have investigated the presence and course of only one or two inguinal nerves [8, 9, 19, 27, 39, 42, 47, 50, 51, 53, 62].

From all these studies it is possible to conclude that, irrespective of course of these nerves individually, the ilioinguinal and iliohypogastric nerve and genital branch are present at a mean of 96, 94 and 90\%, respectively (LE 3b; GR “B”). However, this does not imply that they also are identifiable during open hernia repair. For example, in the study by Rab et al. [55], the ilioinguinal nerve was incorporated in the genitofemoral nerve, lateral to the deep inguinal ring in $44 \%$ of cases. Moosman et al. [45] report the same course in $35 \%$ of patients. Furthermore, these percentages account for the nerves individually, irrespective of the presence and course of the other nerves.

Therefore, according to literature data, the working group estimates that in $70-90 \%$ of cases it is possible to identify all three inguinal nerves as three single nerves (LE 5; GR "D"), while the international audience deemed that it is possible to identify all three nerves in only $40 \%$ of cases, reflecting daily activity, at the present time, in operating rooms worldwide.

Are the nerves covered by an investing fascia or are they naked without a protective fascia to act as a barrier against the mesh?

The ilioinguinal nerve arises from the 12th thoracic and first lumbar nerves. It enters the groin through the trasversus abdominis muscle, medial to the anterior superior iliac spine, and here it is located over the spermatic cord (infrequently under the cremasteric muscle), covered and protected from the mesh and perineural scaring by the investing fascia of the internal oblique muscle.

The iliohypogastric nerve also arises from the 12th thoracic and first lumbar nerves. It enters the groin through the transversus abdominis muscle. It is located between the 
external and internal oblique layers covered and protected by the investing fascia of the internal oblique muscle. This nerve is easily visualized by separating these two layers. It has an easily visible part over the internal oblique aponeurosis and a hidden part within the internal oblique muscle. In fewer than 5\% of patients the iliohypogastric nerve is under the internal oblique aponeurosis in the inguinal region. During repair of hernias in these patients, the subaponeurotic course of the nerve must be determined by noting the location of its exit at the small point of attachment between the internal and external oblique aponeurosis. The surgeon should avoid placing suture or staple below the above mentioned point because the course of the nerve under the internal oblique aponeurosis is inferiorly and laterally [14, 64]. Furthermore, Amid reports that the intramuscular segment of the nerve runs along the lower edge of the internal oblique muscle in $10 \%$ of 210 patients who have undergone extended triple neurectomy [14].

Additionally, adipose connective tissue may be interposed, filling musculoaponeurotic deficiencies in the internal oblique muscle that are present in approximately $48 \%$ of patients [19].

The genital branch of the genitofemoral nerve, originating from the first and second lumbar nerves, enters the groin through the deep ring. It is located under the cord, next to the external spermatic vessels, covered by the deep cremasteric fascia and cremasteric muscle that is derived from the fibres of the internal oblique and trasversus abdominis muscle. The main trunk of the genitofemoral nerve, the preperitoneal segment of its genital branch as well as its femoral branch, are located in the parietal compartment of the preperitoneal space and have no fascial coverage to protect them from direct contact with mesh.

Although it is generally believed to be difficult to see this small nerve, its location can be easily determined by the so called visible "blue line" [18], consisting of the external spermatic vein that is always adjacent to the nerve. It is not actually necessary to always identify the nerve, but it is important to know where is located and to save the external spermatic vessels, reserving its identification only in case of bleeding of the external spermatic vessels or in case of cremasteric muscle resection.

Both the working group and the international audience concluded that ilioinguinal and iliohypogastric nerves are both covered by the investing fascia of internal oblique muscle, and that the inguinal segment of the genital branch is covered by the deep cremasteric fascia. In fact, between the external oblique aponeurosis and the internal oblique muscle lies a layer of areolar connective tissue.
Could the covering fascia be damaged, or is it advisable to protect it?

There are initial experimental data that direct contact of mesh with nerves leads to neuropathy due to certain ultrastructural changes in the nerves. These reports are consistent with the preliminary results of ongoing study by Amid into structural changes in nerves in patients suffering from postherniorrhaphy pain (P.K. Amid, unpublished data). Leaving intact the deep cremasteric fascia covering the genital branch of the genitofemoral nerve under the cord, the nerve is protected from perineural scaring and direct contact with mesh.

If the funiculus is luxated from the inguinal floor, the cremasteric fascia encircling the genital branch and cremasteric vessels should be included. To a certain extent this could protect not only the genital branch but also the parasympathic paravasal nerves and other contents of the spermatic cord against future mesh and fibrosis (LE 5; GR “D”).

Both the working group and the international audience deemed that dissection of the upper and lower leaf of the aponeurosis of the external oblique muscle should be conducted as close to the aponeurosis as possible in order to prevent dissection of the areolar and adipose connective tissue layer that form a protective barrier between the nerves and the future mesh or fibrotic tissue (LE 5; GR“D”).

Is the intramuscular segment of the iliohypogastric nerve at risk when a suture is passed through the internal oblique muscle?

According to a Dutch anatomical study, the iliohypogastric nerve runs at a mean of 2.4 (range 1.5-4.4) cm cranially to the internal ring [64] (LE 3b; GR "B").

In $11 \%$ of cases, the iliohypogastric nerve is still intramuscular when coursing cranial to the internal ring, and perforates the internal oblique muscle approximately halfway along, and cranially to, the spermatic cord (LE 3b; GR "B") [64]. The intramuscular segment of the iliohypogastric nerve is the most vulnerable neural structure during open inguinal hernia repairs, passing suture through the internal oblique muscle. During the Lichtenstein repair, fixation (suturing or stapling) of the upper edge of the mesh to the internal oblique muscle instead of its aponeurosis (the so-called conjoint tendon) should be avoided as it can injure the intramuscular portion of the iliohypogastric nerve.

In such cases, ignorance of the course of this nerve exposes this nerve to possible injury. 


\section{Effect of nerve identification on the rate of postherniorraphy chronic pain}

Is there any correlation between identification vs nonidentification of the three inguinal nerves and chronic pain?

Much controversy still exists in the literature regarding which treatment to carry out for the ilioinguinal, iliohypogastric and the genital branch of genitofemoral nerves during hernia repair. The majority of surgeons still do not detect them, as recently confirmed for the iliohypogastric nerve (detected in only $32 \%$ of cases), and for the genital branch of the genitofemoral nerves (detected in only $36 \%$ of cases) [64]. An Italian prospective multicenter study of 973 cases, and a French single center study of 1,332 cases are the only two published studies reporting the results of the role of the identification of all three inguinal nerves (2,305 cases all together) with a long follow-up period (ranging from 1 to 5 years) $[13,35]$. Both studies concluded that identification and preservation of all three inguinal nerves (this was the case in 924 patients considering the two papers together) during open inguinal hernia repairs (with or without mesh) reduces chronic incapacitating groin pain to less than $1 \%$ : the mean incidence of chronic pain was $0.8 \%$ (range $0-1.6 \%$ ). The Italian study also demonstrated that the risk of developing inguinal chronic pain increased with the number of nerves concomitantly undetected (RR 19.2; CI 2.3-157.7; $P<0.006)$. Likewise, the division of nerves was correlated strongly with the presence of chronic pain (RR 28.6; CI 2.6-31.2; $P<0.001)$ [13].

Accordingly, literature data, the working group and the international audience all concluded that the identification and protection of all three inguinal nerves decreases the risk of developing postoperative severe chronic pain.

For all these reasons, we strongly suggest that the identification of all three nerves plays an important role in reducing the risk of post operative chronic pain.

Should the identified nerve be preserved, divided, resected (neurectomy)?

All the published studies report data concerning division or neurectomy versus preservation of only a single nerve (generally the ilioinguinal nerve, probably because it is the easiest to recognize during hernia repair) without giving any data concerning the other two nerves, forgetting that all three nerves contribute to the sensory innervation of the groin [11]. Thus, results from studies with even an apparently high level of evidence grade of recommendation may be distorted because the two nerves not considered could be unintentionally divided or injured during the operation and, for this reason, chronic pain could result [11].
In Ravichandran [57] (LE 2b; GR "B") and Mui [46] (LE 1b; GR “A”) studies (reporting results of preservation versus neurectomy of only the ilioinguinal nerve) the results are also limited by small sample size, with only 20 and 50 patients, respectively, in each arm, leading to low statistical power, and the follow-up at 6 months is too short; also in Dittrik's study [28] (LE 2b; GR “B”) the distribution between the two arms is not homogeneous (66 patients vs 24 patients).

Picchio [54] (LE 1b; GR "A") reports that pain is not affected by elective neurectomy (according to the new nomenclature) of the ilioinguinal nerve, as also reported by Pappalardo [52] for iliohypogastric neurectomy.

Thus, literature data, the working group and the international audience concluded that identification and protection of all three nerves during open inguinal hernia repair (with or without mesh) reduces the risk of chronic incapacitating pain to less than $1 \%$.

Should the identified nerve be isolated or left in its natural bed without manipulation, and should care be taken not to remove the covering fascia of the nerve?

No published data are available regarding manipulation vs no manipulation of the preserved nerve. However, taking anatomical studies into consideration [63], the working group suggests to avoid removing the nerves from their natural bed as much as possible and not to remove the covering fascia (L.E. 5 G.R.D).

Should a suspected injured nerve or a nerve in the way of repair be saved by any means possible or resected?

Current literature concerning this point is inconsistent and opinions differ considerably. Indeed, no published data are available.

Some authors emphasize that when an injury has occurred, the intramuscular portion of the nerve must be resected, and that merely dividing the nerve at the point of its emergence is inadequate $[10,14]$. These data are supported by the increasing success rate of pain relief with extended versus standard neurectomy procedures obtained by Amid [14] for patients with chronic postherniorrhaphy pain (LE 4; GR “C”), and by Alfieri's results [13] reporting that the increased risk of developing chronic pain with the number of nerves divided could be explained by the fact that resection of the unidentified nerve has generally been performed distal to its origin, leaving the site of the injured nerve intact to continue to generate a pain signal and exposing it to neuroma formation (LE 5; GR "D"). At the same time, there is no scientific evidence that the cut end of the nerve should be left exposed or buried in the muscle. However, during the consensus conference, the working 
group and the international audience agreed to recommend to completely remove a suspected injured nerve or a nerve in the way of repair, and implant the proximal cut end in the muscle, waiting for definitive results from literature (L.E. 5 G.R.D).

Should the cut ends of the nerve be left alone, ligated, or cauterized?

No published data are available concerning the treatment of cut ends of nerves and, consequently, it is impossible to suggest any scientific recommendation. This aspect was well evident from the contrasting opinions of the participants attending the international audience session.

How can the inguinal segment of the genital branch of genitofemoral nerve be identified and protected during the open procedure?

Identifying and protecting the inguinal segment of the genital branch nerve during hernia repair is not a difficult step. It can be recognized by identifying and keeping the external spermatic vein (the so-called "blue line") with the cord. However the genital branch nerve can be damaged if inadvertently sectioned, entrapped, or secured, for example if a continuous suture is introduced along the inguinal ligament, or injured if the external spermatic vessels are divided to skeletonize the cord $[10,15]$ without its identification.

Should the cremasteric layer be saved or resected?

No published data are available in the literature. However, the working group suggests that it is advisable for hernia mesh repair to save the cremasteric layer in order to reduce the risk of ilioinguinal and genital branch nerve damage (L.E. 5; G.R.D).

\section{Effect of glue versus suture on the rate of chronic pain after Lichtenstein repair}

At present in the literature there is no high level of evidence on the effect of glue vs suture on the rate of chronic pain after Lichtenstein repair: two prospective studies [2124] and three controlled clinical trials [25-33] (L.E. 2B; G.R.B) report a reduced rate of chronic pain (the lowest is $4.5 \%)$.

Boyer [21] presented a rate of $4.3 \%$ of chronic pain in a prospective open clinical trial with 350 patients who underwent Lichtenstein repair with fixation of the mesh with fibrin sealant.
Canonico reported no late complications in 80 patients enrolled in a prospective observation study at 1 year after surgery [24].

In a prospective randomized clinical trial with 46 patients enrolled, Helbling reported a reduction in the rate of acute pain at 3 months after surgery from $12.5 \%$ in the suture group to $4.5 \%$ in the fibrin sealant group [33]. Again, in a prospective control trial, Castaldi reported a reduced rate of acute pain $(11.4 \%)$ in a fibrin sealant group versus a control group (37.1\%) at 1 month after surgery [25]. Hidalgo reported 55 patients who underwent bilateral inguinal hernia repair in one operation with the Lichtenstein technique: on the right side the mesh was fixed with polypropylene stiches; on the left side fibrin glue was used. Pain was more often present and more frequent on the right side, although tolerable in all cases [34].

The final results of another, multicenter, prospective, controlled, blinded, randomized, study to evaluate pain and further disabling complications in patients undergoing Lichtenstein technique for primary inguinal hernia repair by fixing the mesh with fibrin sealant versus sutures are awaited.

When glue is used, should we still identify and protect the nerves?

No data are present in the literature regarding whether nerves should be identified and protected even if glue is used. However, the working group and a large percentage of the international audience $(82.5 \%)$ agreed that nerves should be identified and protected in any case (L.E. 5; G.R.D).

\section{Prophylactic neurectomy to reduce the rate of post herniorrhaphy chronic pain}

Is there convincing evidence that prophylactic division or resection of nerves during hernia repair will reduce the rate of chronic pain?

Three randomized trials have examined the effect of prophylactic neurectomy on chronic pain, [46, 54, 57]. In all three trials, the ilioinguinal nerve was either divided or partially resected, and patients were assessed for pain, numbness and touch and pain sensation at 1, 6 and 12 months. None of these studies found differences in pain or numbness, although one [57] found significant improvements in activities such as cycling and walking in favor of the nerve division group. In a prospective study of 973 patients undergoing inguinal hernia repair where all nerves were identified, nerve division was associated with an increased risk of moderate to severe pain [13]. 
The working group, in agreement with the published results in literature, agreed to conclude that there is no evidence that prophylactic neurectomy reduces chronic pain after inguinal hernia repair. On the contrary, indirect evidence suggests that identification and preservation of all nerves may be beneficial $[13,17,35]$. However, further studies addressing this issue are required.

\section{Surgical treatment of chronic groin and testicular pain}

When to consider medical pain management treatment versus surgical treatment?

Initial acute postoperative pain treatment should be as effective as possible and standard pharmacological pain treatment (gabapentanoids, tricyclics, etc.) [30] for neuropathic pain should be instituted earlier in patients with severe pain. The question of whether this may reduce development of chronic pain is debatable in the absence of any conclusive data [22]. Likewise, there are no conclusive data in literature on whether a switch from medical treatment to surgical treatment in the management of chronic groin post-operative pain is effective.

The working group decided to consider reasonable surgical treatment only after 1 year postoperatively, when the inflammatory response has decreased, and only when pain intensity curtails activity and conventional treatment has failed (L.E. 5, G.R.D). However, a multi-center protocol collaboration is required to define indications for surgery vs medical treatment approaches.

Finally, we need data on how to treat patients with immediate very severe postoperative pain of a neuropathic pain type, suggesting nerve entrapment either by sutures, clips or tacs, and where acute re-exploration with alleviation of the causative factor may be indicated.

Should surgical treatment of chronic neuropathic pain include all three nerves?

Triple neurectomy is reported to be a proven surgical treatment for chronic postherniorrhaphy pain intractable to multidisciplinary pain management, with a success rate of operation ranging from 80 [41] to $95 \%[14,59]$, while neurolysis has been demonstrated not to be a viable surgical procedure.

Triple neurectomy should be reserved for those patients who do not respond to non-surgical pain management treatment, but were pain-free prior to their original hernia repair, or if they did have pain, their post-operative pain was different from their pre-operative pain, and has the characteristic features of neuropathic pain.
In agreement with the three major published studies cited above, the working group and the international audience recommend that, when indicated, surgical treatment of chronic neuropathic pain should include all three nerves.

The surgical treatment of pain may pose different problems after placement of mesh in the parietal compartment of the preperitoneal space (during both open and laparoscopic hernia repair). In fact, during laparoscopic hernia repair, nerves in front of the trasversalis fascia are at risk of entrapment by fixating devices [14]. As explained above, behind the trasversalis fascia, the main trunk of genitofemoral nerve, the preperitoneal segment of its genital branch as well as its femoral branch, which are located in the parietal compartment of the preperitoneal space, have no fascial coverage to protect them from direct contact with the mesh. Moreover, the nerves within the preperitoneal space are not easily accessible during operative exploration and, consequently, surgical treatment of inguinodynia and orchalgia after preperitoneal hernia repair is less likely to improve the patient's symptoms. Possible treatment for neuropathy following open and laparoscopic preperitoneal repair is proposed by transabdominal or extraperitoneal approach to the retroperitoneal space for transaction of peri-inguinal nerve over the psoas muscle [16].

Should the intramuscular segment of iliohypogastric be included in triple neurectomy?

Recent observations of groin neuroanatomy have prompted a modification of Amid's neurectomy technique to include a more extensive resection of the iliohypogastric nerve and, for patients with orchalgia, resection of nerves within the lamina propria of the vas deferens as well.

Amid reports to have improved the outcome of tripe neurectomy in 210 patients, resecting the intramuscular portion of the iliohypogastric nerve, instead of cutting the nerve at the point of its emergence from the internal oblique muscle. The nerve should be followed and severed proximal to the surgical field of the original hernia repair. Although a firm conclusion should not be drawn from such a small number of cases, Amid also reports that resection of the paravasal nerves seems to be a useful addition to a triple neurectomy for patients with orchalgia associated with inguinodynia, to eliminate testicular pain [14].

Consequently, the working group suggest including the intramuscular segment of the iliohypogastric nerve during triple neurectomy operation, even at the lowest LE and GR (LE 5; GR D). 
Method (physical examination vs telephone) and length of follow up

In order to provide new and improved knowledge on the development of persistent postherniotomy pain, we need to have large consecutive series with continuous follow up including physical examination and neurophysiological assessment [2] initially about every 6 months, and annually after about 2 years. Such examinations should also include preoperative characteristics of the patient (nociceptive functions, pain genes, psycho-social factors, pain in other parts of the body, etc.) [32, 37] and detailed intraoperative descriptions of nerve handling and placement of suture/clips/mesh, when appropriate. The working group suggests physical examination, including a quantitative sensory test (QST). Follow up is indefinite (natural course).

\section{Conclusions}

Although chronic pain following inguinal hernia repair is a well known complication, much confusion still exists regarding definitions, terminology, neuroanatomy, and its prevention and treatment.

A lot of controversy also still exists with regard to the role of inguinal nerves in the genesis of post-herniorrhaphy inguinodynia and orchalgia, as well as the treatment of the three inguinal nerves, the role of glue during inguinal hernia repair, and the treatment of groin chronic pain.

In the present study, we report the results of an international consensus conference held in Rome in 2008, to which an expert group of nine people (working group) and an international audience of 200 people took part.

Here, we provide the terminology and definitions to be used in the field of groin chronic pain, prepared by the international consensus conference, and some guidelines, valid at the present time, for some of the above-mentioned problems; others remain open questions.

- The incidence of debilitating chronic pain after any form of open or laparoscopic repair affecting normal daily activities or work has been estimated to be from 0.5 to $6.0 \%$.

- Post-herniorraphy pain was defined as pain arising as a direct consequence of a nerve lesion or a disease affecting the somatosensory system, in patients who did not have groin pain before the original operation or, if they did, the post-operative pain was different from their pre-operative pain. The working group suggests to modify the IASP definition [26] to include only chronic pain that is present from 3 months after surgery and which lasts beyond 6 months after surgery.
- The clinical diagnosis of neuropathic pain cannot be defined at present.

- The management of inguinal nerves during a hernia operation must be described in the operative notes using the following terminology: cutting or dividing a nerve means interrupting the continuation of a nerve; resection of a nerve or neurectomy means removing a segment of a nerve along the inguinal canal. It is appropriate to mention the handling of the cut ends (ligation, cauterization or nothing) and the proximal and distal level of section.

- The international audience results indicated that it is possible to identify the ilioinguinal, iliohypogastric and genital branch of genitofemoral nerves in only $40 \%$ of cases, reflecting the daily practise in operating rooms worldwide, where the majority of surgeons continue not to detect them. On the contrary, the working group, in agreement with literature data, estimates that it is possible to identify all three nerves as single nerves in $70-90 \%$ of patients.

- Both the working group and the international audience agreed to recommend to identify and preserve all three inguinal nerves, because, according to current literature data, this seems to reduce the risk of post-operative chronic pain; however, if a nerve is in the way of a repair or is suspected to have been injured during the operation, the consensus view was to recommend to completely remove it, but never simply cut the nerve leaving the two stumps of nerve in the surgical field. However, no scientific recommendation is possible regarding the treatment of the cut ends of the nerve, or on resectioning or preserving the cremasteric layer.

- No definitive results on the effect of glue versus suture on the rate of chronic pain are available.

- The working group agreed to recommend medical pain management as the first choice in the treatment of postoperative chronic pain. If medical treatment has failed for more than 1 year after operation, and if pain intensity interferes with normal daily activities, then triple neurectomy is indicated. This operation should be performed only by experienced hands.

Conflict of interest No conflicts of interest.

\section{References}

1. Aasvang EK, Bay-Nielsen M, Kehlet H (2006) Pain and functional impairment 6 years after inguinal herniorrhaphy. Hernia 10:316-321

2. Aasvang EK, Brandsborg B, Christensen B, Jensen TS, Kehlet H (2008) Neurophysiological characterization of postherniorrhaphy pain. Pain 137:173-181 
3. Aasvang EK, Hansen JB, Kehlet H (2009) Pre-operative pain and sensory function in groin hernia. Eur J Pain 13:1018-1022

4. Aasvang EK, Kehlet H (2010) Persistent sensory dysfunction in pain-free herniotomy patients. Acta Anaesthesiol Scand 54:291-298

5. Aasvang E, Kehlet H (2005) Surgical management of chronic pain after inguinal hernia repair. Br J Surg 92:795-801

6. Aasvang EK, Kehlet H (2009) The effect of mesh removal and selective neurectomy on persistent postherniotomy pain. Ann Surg 249:327-334

7. Aasvang EK, Mohl B, Bay-Nielsen M, Kehlet H (2006) Pain related sexual dysfunction after inguinale herniorrhaphy. Pain 122:258-263

8. Akita K, Niga S, Yamato Y, Muneta T, Sato T (1999) Anatomic basis of chronic groin pain with special reference to sports hernia. Surg Radiol Anat 21(1):1-5

9. Al-dabbagh AK (2002) Anatomical variations of the inguinal nerves and risks of injury in 110 hernia repairs. Surg Radiol Anat 24(2):102-107

10. Alfieri S (2008) A met-analytic approach to ilioinguinal nerve. Excision or preservation during open inguinal hernia repair. Ann Surg 247(6):1078-1080

11. Alfieri S, Di Miceli D, Doglietto GB (2007) Prophylactic ilioinguinal neurectomy in open inguinal hernia repair. Ann Surg 245(4):663

12. Alfieri S, Di Miceli D, Doglietto GB (2007) Re: nerve management during open hernia repair. Br J Surg 94(7):914

13. Alfieri S, Rotondi F, Di Giorgio A, Fumagalli U, Salzano A, Di Miceli D, Ridolfini MP, Sgagari A, Doglietto G, Group Groin Pain Trial (2006) Groin pain trial group. Influence of preservation versus division of ilioinguinal, iliohypogastric, and genital nerves during open mesh herniorrhaphy: prospective multicentric study of chronic pain. Ann Surg 243(4):553-558

14. Amid PK (2002) A 1-stage surgical treatment for postherniorrhaphy neuropatic pain: triple neurectomy and proximal end implantation with mobilization of the cord. Arch Surg 137:100-104

15. Amid PK (2004) Causes, prevention, and surgical treatment of postherniorrhaphy neuropathic inguinodynia: triple nerurectomy with proximal end implantation. Hernia 8(4):343-349

16. Amid PK, Hiatt JR (2008) Surgical anatomy of the preperitoneal space. J Am Coll Surg 207(2):295 (author reply 295-296)

17. Amid PK, Hiatt JR (2007) New understanding of the causes and surgical treatment of postherniorrhaphy inguinodynia and orchalgia. J Am Coll Surg 205(2):381-385

18. Amid PK (2004) Lichtenstein tension-free hernioplasty: its inception, evolution, and principles. Hernia 8(1):1-7

19. Anson BJ, Morgan EH, Mc VC (1960) Surgical anatomy of the inguinal region based upon a study of 500 body-halves. Surg Gynecol Obstet 111:707-725

20. Bay-Nielsen M, Perkins FM, Kehlet H (2001) Pain and functional impairment 1 year after inguinal herniorrhaphy: a nationwide questionnaire study. Ann Surg 233:1-7

21. Boyer (2004) Oral presentation at 26th Congress of the EHS in 2004

22. Brennan TJ, Kehlet H (2005) Preventive analgesia to reduce wound hyperalgesia and persistent postsurgical pain: not an easy path. Anesthesiology 103:681-683

23. Callesen T, Bech K, Kehlet H (1999) Prospective study of chronic pain after groin hernia repair. Br J Surg 86:1528-1531

24. Canonico S, Santoriello A, Campitiello F, Fattopace A, Corte AD, Sordelli I, Benevento R (2005) Mesh fixation with human fibrin glue (Tissucol) in open tension-free inguinal hernia repair: a preliminary report. Hernia 9:330-333

25. Castaldi M (2004) The use of fibrin glue in open inguinal hernia, abstract of the 26th congress of the EHS in 2004
26. Classification of chronic pain (1986) Description of chronic pain syndromes and definitions of pain terms. Prepared by the International Association for the study of pain Subcommitte on Taxonomy. Pain Suppl 3:S1-S226

27. Diop M, Dia A, Ndiaye A, Lo EA, Sow ML, Ndiaye PD (2000) Emergence and course of the ilioinguinal nerve of the groin. Morphologie 84(266):29-32

28. Dittrick GW, Ridl K, Kuhn JA, McCarty TM (2004) Routine ilioinguinal nerve excision in inhuinal hernia repairs. Am J Surg 188(6):736-740

29. Ferzli GS, Edwards ED, Khoury GE (2007) Chronic pain after inguinal herniorrhaphy. J Am Coll Surg 205:333-341

30. Finnerup NB, Otto M, McQuay HJ, Jensen TS, Sindrup SH (2005) Algorithm for neuropathic pain treatment: an evidence based proposal. Pain 118:289-305

31. Franneby U, Gunnarsson U, Andresson M, Heuman R, Nordin P, Nyrén O, Sandblom G (2008) Validation of an inguinal pain questionnaire for assessment of chronic pain after groin hernia repair. Br J Surg 95:488-493

32. Haapaniemi S, Nilsson E (2002) Recurrence and pain three years after groin hernia repair. Validation of postal questionnaire and selective physical examination as a method of follow-up. Eur $\mathbf{J}$ Surg 168:22-28

33. Helbling C, Schlumpf R (2003) Sutureless Lichtenstein: first results of a prospective randomised clinical trial. Hernia $7(2): 80-84$

34. Hidalgo M, Castillo MJ, Eymar JL, Hidalgo A (2005) Lichtenstein inguinal hernioplasty: sutures versus glue. Hernia 9(3):242244

35. Izard G, Gailleton R, Randrianasolo S, Houry R (1996) Treatment of inguinal hernia by McVay's technique. A propos of 1332 cases. Ann Chir 50:775-776

36. Kehlet H (2008) Chronic pain after groin hernia repair. Br J Surg 95:135-136

37. Kehlet H, Jensen TS, Woolf CJ (2006) Persistent postsurgical pain: risk factors and prevention. Lancet 367:1618-1625

38. Lichtenstein IL, Shulman AG, Amid PK, Montllor MM (1998) Causes and prevention of postherniorrhaphy neuralgia: a proposed protocol for treatment. Am J Sur 155:786-790

39. Liu WC, Chen TH, Shyu JF, Chen CH, Shih C, Wang JJ, Kung SP, Lui WY, Wu CW, Liu JC (2002) Applied anatomy of the genital branch of the genitofemoral nerve in open inguinal herniorrhaphy. Eur J Surg 168(3):145-149

40. Loos MJ, Roumen RM, Scheltinga MR (2007) Classifying postherniorrhaphy pain syndromes following elective inguinal hernia repair. World J Surg 31:1760-1765

41. Madura JA, Madura JA 2nd, Copper CM, Worth RM (2005) Inguinal neurectomy for inguinal nerve entrapment; an experience with 100 patients. Am J Surg 189:283-287

42. Mandelkow H, Loeweneck $H$ (1988) The iliohypogastric and ilioinguinal nerves. Distribution in the abdominal wall, danger areas in surgical incisions in the inguinal and pubic regions and reflected visceral pain in their dermatomes. Surg Radiol Anat 10(2): 145-149

43. McCarthy M Jr, Jonasson O, Chang CH, Pickard AS, GiobbieHurder A, Gibbs J, Edelman P, Fitzgibbons R, Neumayer L (2005) Assessment of patient functional status after surgery. J Am Coll Surg 201:171-178

44. Mirilas P, Mentessidov A, Shandalakis JE (2008) Secondary internal inguinal ring and associates surgical planes: surgical anatomy, embryology, applications. J Am Coll Surg 2006:561-570

45. Moosman DA, Oelrich TM (1977) Prevention of accidental trauma to the iloinguinal nerve during inguinal hernoirrhaphy. Am J Surg 133(2):146-148

46. Mui WL, Ng CS, Fung TM, Cheung FK, Wong CM, Ma TH, Bn MY, Ng EK (2006) Prophylactic ilioinguinal neurectomy in open 
ilioinguinal repair: a double-blind randomized controlled trial. Ann Surg 244(1):27-33

47. Ndiaye A, Diop M, Ndoye JM, Konaté I, Ndiaye AI, Mané L, Nazarian S, Dia A (2007) Anatomical basis of neuropathies and damage to the ilioinguinal nerve during repairs of groin hernias (about 100 dissections). Surg Radiol Anat 29(8):675-681

48. Nienhuijs S, Staal E, Strobbe L, Rosman C, Groenewoud H, Bleichrodt R (2007) Chronic pain after mesh repair of inguinal hernia: a systematic review. Am J Surg 194:394-400

49. O'Dwyer PJ, Alani A, McConnachie A (2005) Groin hernia repair: postherniorrhaphy pain. World J Surg 29:1062-1065

50. Oelrich TM, Moosman DA (1977) The aberrant course of the cutaneous component of the ilioinguinal nerve. Anat Rec 189(2):233-236

51. Papadopoulos NJ, Katritsis ED (1981) Some observations on the course and relations of the iliohypogastric and ilioinguinal nerves (based on 348 specimens). Anat Anz 149(4):357-364

52. Pappalardo G, Frattaroli FM, Mongardini M, Salvi PF, Lombardi A, Conte AM, Arezzo MF (2007) Neurectomy to prevent persistent pain after inguinal herniorraphy: a prospective study using objective criteria to assess pain. World J Surg 31(5):1081-1086

53. Peschaud F, Malafosse R, Floch-Prigent PL, Coste-See C, Nordlinger B, Delmas V (2006) Anatomical bases of prolonged ilio-inguinal-hypogastric regional anesthesia. Surg Radiol Anat 28(5):511-517

54. Picchio M, Palimento D, Attanasio U, Matarazzo PF, Bambini C, Caliendo A (2004) Randomized controller trial of preservation or elective division of ilioinguinal nerve on open inguinal hernia repair with polypropylene mesh. Arch Surg 139(7):755-758

55. Rab M, Ebmer J, Dellon AL (2001) Anatomic variability of the ilioinguinal and genitofemoral nerve: implications for the treatment of groin pain. Plast Reconstr Surg 108(6):1618-1623

56. Rasmussen PV, Sindrup SH, Jensen TS, Bach FW (2004) Symptoms and signs in patients with suspected neuropathic pain. Pain 110:461-469
57. Ravichandran D, Kalambe BG, Pain JA (2000) Pilot randomized controlled study of preservation or division of ilioinguinal nerve in open mesh repair of inguinal hernia. Br J Surg 87(9): 1166-1167

58. Staal E, Nienhuijs SW, Keemers-Gels ME, Rosman C, Strobbe LJ (2008) The impact of pain on daily activities following open mesh inguinal hernia repair. Hernia 12:153-157

59. Starling JR, Harms BA (1994) Ilioinguinal, iliohypogastric, and genitofemoral neuralgia. In: Bendavid R (eds) Prostheses and abdominal wall hernia. Landes, Austin, pp 3351-3567

60. Treede RD, Jensen TS, Campbell JN, Cruccu G, Dostrovsky JO, Griffin JW, Hansson P, Hughes R, Nurmikko T, Serra J (2008) Neuropathic pain: redefinition and a grading system for clinical and research purposes. Neurology 70:1630-1635

61. van Hanswijck de JP, Lloyd A, Hrsfall L, Tan R, O'Dwyer PJ (2008) The measurement of chronic pain and health-related quality of life following inguinal hernia repair: a review of the literature. Hernia 12:561-569

62. Whiteside JL, Barber MD, Walters MD, Falcone T (2003) Anatomy of ilioinguinal and iliohypogastric nerves in relation to trocar placement and low transverse incisions. Am J Obstet Gynecol 189(6):1574-1578 (discussion 1578)

63. Wijsmuller AR, Lange JF, Kleinrensink GJ, van Geldere D, Simons MP, Huygen FJ, Jeekel J, Lange JF (2007) Nerve-identifying inguinal hernia repair: a surgical anatomical study. World $\mathrm{J}$ Surg 31(2):414-420 (discussion 421-422)

64. Wijsmuller AR, Lange JF, van Geldere D, Simons MP, Kleinrensink GJ, Hop WC, Jeekel J, Lange JF (2007) Surgical techniques preventing chronic pain after Lichtenstein hernia repair: state-of-the-art vs daily practice in the Netherlands. Hernia 11(2):147-151 